\title{
Paternalismus im Strafrecht
}

Günther Jakobs"

Rezension zu Andreas von Hirsch, Ulfried Neumann, Kurt Seelmann (Hrsg.), Paternalismus im Strafrecht. Die Kriminalisierung von selbstschädigendem Verhalten (Studien zur Strafrechtstheorie und Strafrechtsethik, Bd. 1), Baden-Baden (Nomos) 2010, 355 S., brosch. 89,- $€$, ISBN 978-3 8329-5518-2

\section{Vorbemerkungen}

Die Rezension des vorliegenden Sammelbandes, Beiträge zu einer Tagung in Frankfurt am Main 2007, erfordert einige Vorbemerkungen. „Paternalismus“ heißt die Beschränkung der Verhaltensfreiheit einer Person zugunsten ihres Wohls. Beim direkten Paternalismus wird die Person notfalls gezwungen, ein bestimmtes Verhalten nicht zu vollziehen, eventuell wird auch eine Sanktion (Geldbuße, Strafe) angedroht; häufig genannt werden etwa das Autofahren, ohne einen Sicherheitsgurt angelegt zu haben, oder das Motorradfahren ohne Schutzhelm. Beim indirekten Paternalismus wird dritten Personen eine das Wohl des zu Schützenden gefährdende Kooperation mit diesem untersagt; das Panorama reicht von staatlichen Forderungen an die Sicherheit technischer Produkte, gerichtet an Produzenten und Händler, über das Verbot, bestimmte Medikamente ohne ärztliche Verschreibung abzugeben, bis hin zum Verbot der Tötung auf Verlangen.

In jeder entwickelten Gesellschaft gibt es Vorstellungen darüber, was legitimerweise rechtlich geregelt werden, also nicht dem freien Spiel der Kräfte überlassen bleiben soll, etwa die Bedingungen der Sicherheit des Straßenverkehrs. Die Höhe der Sicherheit und des verbleibenden Restrisikos lässt sich nicht willkürlich festsetzen, vielmehr muss sie mit anderen Regelungsbereichen - Lebensmittelsicherheit, Sicherheit der Krankenversorgung etc. - austariert werden; mit anderen Worten, es gilt, einen erträglichen Sicherheitsstandard zu finden. Nun ließe sich ein solcher für der Straßenverkehr leicht erreichen: Erlaubt wird nur Schritttempo, - aber diese radikale Lösung ginge am Zweck des Straßenverkehrs vorbei. Oder, alle irgendwie gefährlichen Straßenanlagen werden entschärft, - aber das wäre unbezahlbar. Es bietet sich an, Machbares zu bündeln: bereichsweise Tempolimitierung, Entschärfung einiger Anlagen etc. und Selbstschutz, eben Gurt- und Helmpflicht. Gewiss, man könnte auf die Pflicht zum Selbstschutz verzichten und es bei einer Obliegenheit belassen, also den Leichtsinnigen „billiger“ retten, seine Rente kürzen etc., aber damit ließe sich ganz abgesehen von der Frage, ob so etwas „verwaltbar“ wäre - das Problem nicht erledigen; denn die Institution „Straßenverkehr“ bliebe mit dem Makel belastet, zu

* Prof.Dr. Dr. h.c. mult. Günther Jakobs ist emeritierter Professor für Strafrecht und Rechtsphilosophie an der Rheinischen Friedrich-Wilhelms-Universität in Bonn. 
viel „Blut“ zu kosten, mehr noch, den starken Personen mit Überblick und Entscheidungskraft, die begreifen, dass Obliegenheitsverletzungen etwas „kosten“ werden, und bereit sind, die Konsequenzen auf sich zu nehmen, wäre entgegenzuhalten: „Sehet zu, dass diese eure Freiheit nicht gerate zu einem Anstoß der Schwachen “ (1. Kor. $8,9)$.

Bei der Gurt- und Helmpflicht sowie den entsprechenden indirekten Regelungen etwa dem Verbot, Kraftfahrzeuge mit scharfkantigen Inneneinrichtungen zu produzieren und zu vertreiben - geht es also um einen allenfalls mittelbaren Paternalismus: ${ }^{1}$ Die Förderung des Wohls durch Beschneidung der Freiheit dient nicht dem Wohl als Endziel, sondern als Durchgang zum Ziel der „Akzeptation des Regelungskomplexes“.

Noch ein Weiteres kommt hinzu: So wie eine Person, die ihre eigenen Güter verschleudert, in der Regel nicht erwarten kann, dass man ihr gehörigen Respekt vor den Gütern anderer zutraut, so muss auch eine Gesellschaft darauf achten, die Bedingungen des Respekts zu erhalten, und dazu gehört eben auch ein kleiner Aufwand für den Selbstschutz (und nur um einen solchen kleinen Aufwand geht es an dieser Stelle). Die Normen, die diesen kleinen Aufwand vorschreiben, werden ja auch nicht absolutistisch verordnet, sondern unmittelbar oder mittelbar demokratisch beschlossen, und deshalb lassen sie sich in der Regel - es gibt freilich auch einige Übertreibungen - als Selbstbindung der Bürger begreifen, nicht als väterlich gemeinte Bindungen durch einen vorschreibenden Staat.

\section{Kritischer Bericht}

1.) Der soeben thematisierte Schutz von Institutionen durch eine Verpflichtung zum Selbstschutz wird zwar von den Autoren nur vereinzelt behandelt (Kleinig, Australien, S. 149 f.; von der Pfordten, S. 197), aber es fällt auf, dass im Bereich des Verbraucherschutzes und der Gurt- und Helmpflicht bußgeldbewehrte Normen in der Regel recht großzügig legitimiert werden (Husak, USA, S. 129 ff; von der Pfordten, S. 196; Seelmann, S. 251; kritischer freilich Peršak, Slowenien, S.173 ff.). Das dabei mehrfach verwendete Argument, ein Verstoß etwa gegen die Gurtpflicht zeuge allemal von einem kognitiven Defizit (Simester, Singapore, S.270; Schünemann, S. 239 f.), dürfte falsch sein (so auch Peršak, S. 180): Nicht die Risikokenntnis fehlt, vielmehr ist die Risikoscheu unterentwickelt, und zudem dürfte zu bezweifeln sein, ob eine durch nahezu jedes Exemplar einer Tageszeitung widerlegbare Fehleinschätzung, so sie denn vorläge, die Selbstverantwortung des offenbar Desinteressierten ausschlösse. - Das Argument Schünemanns, kognitive Mängel bei der Beurteilung

1 Diese nur vermittelnde Pflicht zum Selbstschutz wird teils aus dem Begriff des Paternalismus ausgeklammert; etwa Hermann, Paternalismus, staatlicher, in: Gosepath u. a. (Hrsg.), Handbuch der politischen Philosophie und Sozialphilosophie, Bd. 2, 2008, S. 962 ff. 
einer Selbstschädigung dürfe der Staat durch „Zwangsaufklärung als soziale Maßnahme" ausräumen (und dem gegenüber sei eine Geldbuße eine geringere Belastung; a. a. O.), passt nicht zu der ansonsten zur Schau getragenen Liberalität.

2.) Heikel steht es um die Legitimität von Verboten, die eigene Würde wegzuwerfen; Stichworte sind „Peepshows“ (wobei erstaunlicherweise nur von der Selbsterniedrigung der Frau die Rede ist), „Gladiatorenkämpfe“, „Selbstversklavung“. Nach Kleinig soll zwar allein wegen eines solchen Verhaltens ein paternalistischer Eingriff nicht zu rechtfertigen sein, wohl aber dann, wenn es zu unumkehrbaren schweren Folgen führt (S. 164; überhaupt gegen die Bestrafung jeglicher Selbsterniedrigung Kasachkoff, Israel, S. 235 ff.), also nicht bei Peepshows (S. 168), jedoch bei Gladiatorenkämpfen und Selbstversklavung (S. 164, 170). Als Kern seiner Begründung formuliert Kleinig nach Auseinandersetzungen mit J. S. Mill, den er nicht radikal-liberal verstehen will (S. 157), und T. H. Green: „Menschliche Freiheit kann nur in einem wohlgeordneten sozialen Umfeld gelebt werden“ (S. 160), so dass neben die Würde des Einzelnen die Würde als „soziale(r) Wert“ trete (S. 146). - Seelmann wendet sich gegen eine Argumentation zugunsten einer „Würde der Gattung“ (S. $241 \mathrm{ff}$.), will aber rechtliche Regelungen zulassen, „wenn der einzelne durch ein bestimmtes Verhalten anderer seine generelle Kompetenz, sich in der Welt zurecht zu finden, einbüßt, wenn er also seiner Orientierungskompetenz verlustig geht" (S.250). Grund der Reglementierungen ist dann die Unverträglichkeit des Verhaltens mit der „Rechtssubjektivität" der anderen (S.252).

Gegen den Versuch, die Klippe der Kollision von Freiheit und Wohl mit der Annahme einer Rechtspflicht gegen sich selbst zu umschiffen, wendet sich Vossenkubl (S. 275 ff., hauptsächlich zur Pflicht, sich nicht selbst zu töten, ${ }^{2}$ aber auch zur Selbsterniedrigung, etwa durch Selbstversklavung, S. 281). Er trennt - im Rahmen der praktischen Philosophie Kants - scharf zwischen einer „Theorie des reinen Willens“, aus dem ein (Selbst-) Instrumentalisierungsverbot als Tugendpflicht folge, und der Handlungsfreiheit, die allein Gegenstand rechtlicher Regelungen sein könne (S. $282 \mathrm{f}$.). „Ein rechtliches Verbot der Selbstverfügung formuliert Kant... nicht“ (S. 284). - Seelmann argumentiert vergleichbar: Rechtspflichten gegen sich selbst, im 18. Jahrhundert geläufig (Chr. Wolff!), würden bei Kant zu Tugendpflichten, und der Würdebegriff sei ein solcher der Tugendlehre. Mit dem im Grundgesetz verwendeten Würdebegriff als Rechtsbegriff verbinde sich deshalb die Gefahr einer Ethisierung des Rechts (S. 242 f.).

3.) Einen Schwerpunkt des Bandes bildet, wie zu erwarten, die Behandlung der Tötung auf Verlangen ( $\$ 216$ StGB), wobei die Rechtslage in Deutschland komplizierter ist als die international geläufige, da die Beteiligung an einer Selbsttötung straffrei,

2 Für eine solche Pflicht Köhler, Die Rechtspflicht gegen sich selbst, in: Jahrbuch für Recht und Ethik, 14 (2006), S. $425 \mathrm{ff}$. 
ja sogar unverboten bleibt. Die Problematik beginnt bereits bei den Versuchen, die Vorschrift einzuordnen: Geht es um eine wegen des Verlangens privilegierte Fremdtötung oder um eine vom Verlangenden durch fremde Hand arrangierte Selbsttötung? Ersteres vertritt mit Nachdruck von der Pfordten mit der Begründung, es sei bei der „äußere(n) Tathandlung als entscheidende(m) Gesichtspunkt für die strafrechtliche Bewertung“ anzusetzen (S.197f.): „Das moderne Strafrecht ist ein Tatstrafrecht und kein Interessenverletzungsstrafrecht" (S. 198). Allerdings müsste bei dieser Sicht begründet werden, warum die Tat mit dem sichtbaren Handlungsvollzug, dem Phänotyp, gleichzusetzen sein soll; etwa bei einer konsentierten Flugreise handelt es sich ja auch nicht um eine bis zur Straffreiheit privilegierte Entführung, ganz zu schweigen von einer Sachbeschädigung auf Verlangen. Die letztere Sicht verfolgen von Hirsch (Großbritannien) und Neumann: „Wenn man das Recht hat, sein Leben zu beenden, sollte man auch das Recht haben, zu entscheiden, wie das geschehen soll“ (S. 81). Mit anderen Worten, sich auf Verlangen töten zu lassen, ist eine Weise, „sein Leben zu beenden“, also eine Selbsttötung durch fremde Hand. $^{3}$

Die unterschiedlichen Ansätze führen zu unterschiedlichen Argumentationslasten. Ist die Tötung auf Verlangen eine privilegierte Fremdtötung, bleibt nur noch die Aufgabe, das Maß der Privilegierung zu bestimmen; jedenfalls kann es keine tatbestandlichen Ausnahmen von der Strafbarkeit geben. So erkennt von der Pfordten zwar an, dass durch das Verbot ,in sehr seltenen Fällen“ einem Lebensmüden jede Gelegenheit genommen wird, aus dem Leben zu scheiden (S. 200), wobei er die Seltenheit durchaus plausibel dartut: Es wird sich bei den Verlangenden fast immer um moribunde Personen handeln, die ihren Tod durch das verbindliche (!) Verlangen nach einem Behandlungsabbruch herbeiführen können (S. 199 f.). Ist das ausnahmsweise nicht der Fall, soll es bei dem Verbot bleiben: „Die Menschen in einer Gesellschaft können sich... legitim dafür entscheiden, die so außerordentlich problematische, eine humanistische Gesellschaft schwer beeinträchtigende Fremdtötung zu untersagen “ (S. 200), dies mit der Konsequenz, dass „man... nicht erwarten“ kann, „jede Form der eigenen Selbstbestimmung durch das Tun anderer realisieren zu können“ (S. 201).

Gegenläufig argumentieren von Hirsch und Neumann. Da die Tötung auf Verlangen nach ihrer Ansicht eine Ausführungsart der Selbsttötung ist, bedarf es besonderer Gründe, sie zu verbieten oder gar zu bestrafen, und die Autoren erörtern das Risiko eines mangelhaften, insbesondere vorschnellen Verlangens, den Wegfall der (vielleicht) vor einem Suicid stehenden „Hemmschwelle“ (S. 72 ff.) und die gegebenenfalls dürftige und nicht überzeugende Begründung des Verlangens (S. 82 ff.). - Dazu

3 So auch Jakobs Tötung auf Verlangen, Euthanasie und Strafrechtsystem, 1998, S. 14 ff.; eingehend zu den zu $\$ 216$ StGB vertretenen Lehrmeinungen Frank Müller, $\mathbb{2} 216$ StGB als Verbot abstrakter Gefährdung. Versuch der Apologie einer Strafnorm, 2010, S. 29 ff., 103 ff. 
einige Bemerkungen: Die genannten Mängel des Verlangens mögen im Einzelfall gegeben sein, können aber auch fehlen, und im letzteren Fall betrifft das Verbot der Tötung auf Verlangen ein abstrakt gefährliches Verhalten, nicht aber ein Verletzungsunrecht. Mit anderen Worten, wenn ein gültiges Verlangen vorliegt, geht es bei der Tötung auf Verlangen nicht um Tötungsunrecht, da die verlangende Person nicht vergewaltigt wird, sondern ihrerseits den Zweckzusammenhang bestimmt; ist das Verlangen aber ungültig, so liegt Totschlagsunrecht vor. Die beiden Autoren nehmen bei einem nicht überzeugend begründeten Verlangen an, es sei „respektlos“, ihm zu entsprechen (S. 82 f. und passim); da aber auch in einem solchen Fall der Verlangende den Zweckzusammenhang bestimmt, lässt sich ein Fremdverletzungsunrecht nicht dartun.

Im Ergebnis plädieren von Hirsch und Neumann für eine „partielle Entkriminalisierung " der Tötung auf Verlangen, und zwar für diejenigen Fälle, in denen eine "zweistufig“ vorzunehmende „Lebensstandardanalyse“ zu dem Ergebnis führe, es mangele an „Lebensqualität“ (S. 89 ff.). Auf der ersten Stufe soll zu prüfen sein, „ob die Person unter einer ernsthaften Beeinträchtigung der Ressourcen und Fähigkeiten leidet, die unter normalen Umständen für ein Minimum an Lebensqualität erforderlich sind“ (S. 91). Auf der zweiten Stufe sollen die „persönlichen Präferenzen“ berücksichtigt werden (S.92), dies, wenn der Rezensent es recht sieht, jedoch nur zur Akzeptation oder Verwerfung des positiven Ergebnisses der ersten Stufe (Die schwere Beeinträchtigung ist gegeben!), nicht aber um ein negatives Ergebnis zu überspielen. Die Analyse soll „prozedural“ gesichert werden (S. 77 ff., 96), insbesondere zur Garantie der Einsichtsfähigkeit des Verlangenden und der Vollzugsreife seines Wunsches. Die skizzierte Entkriminalisierung dürfte nach der Ansicht der beiden Autoren „erheblich weiter" reichen, als das bei den geläufigen Vorschlägen für Fälle medizinischer Hoffnungslosigkeit der Fall ist: Weder der Beginn der Sterbephase noch akuter Schmerz noch eine bevorstehende Verschlechterung des Zustands sollen conditiones sine quae non bilden.

Trotz noch zu formulierender Bedenken überzeugt das Konzept den Rezensenten insoweit, als anerkannt wird, das menschliches Leben heute (auch) medizinisch gemachtes Leben ist. Bei dieser Lage wäre es unaufrichtig, das Lebensende allein dem Walten der Natur überlassen zu wollen, und die durch von Hirsch und Neumann herausgearbeiteten Ausnahmen vom Verbot der Tötung auf Verlangen wirken, so gesehen, noch recht eng. Aber die Ausnahmen müssen, sollen sie nicht entweder nur auf dem Papier stehen oder, im Gegenteil, ausufern, verwaltbar sein. Mag sich diese Verwaltung für die prozedurale Sicherung noch leisten lassen, so doch kaum, was die Berücksichtigung persönlicher Präferenzen angeht: Soviel Einzelfallgerechtigkeit bringt eine Komplexität, die mit rechtlicher garantierter Erwartenssicherheit unverträglich sein könnte. - Es wäre schon viel erreicht, wenn zunächst den ernsthaft 
Sterbewilligen die Gelegenheit geboten würde, sich auf eine sichere und würdige Weise selbst zu töten. Wenn sich dann eine Sterbekultur gebildet hat („Kultur“ im positiven Sinn verstanden), lässt sich auch die Tötung auf Verlangen entkriminalisieren, und zwar viel umfassender, als von Hirsch und Neumann vorschlagen. - Eines ist noch anzumerken: Wenn die Autoren ihren Vorschlag auf Fälle aktuellen, ausdrücklichen Verlangens beschränken wollen (S. 197), so wird ihnen das auf Dauer nicht gelingen; denn sobald sich Standards herausgebildet haben, wäre es schiere Grausamkeit, den zur Äußerung eines Verlangens Unfähigen das zu versagen, was andere geläufig erhalten. Wer daran zweifelt, der führe sich vor Augen, wie längst bei der indirekten Euthanasie verfahren wird.

4.) Hörnle nimmt sich das Verbot der Eizellenspende nach deutschem Recht und die Restriktionen dieser Spende nach den Rechten anderer europäischer Länder vor (S. 111 f.). Ein zu hohes Lebensalter der empfangenden Frau lässt sie als möglichen Verbotsgrund noch durchgehen, nicht aber die Vermeidung einer „gespaltenen Mutterschaft“: Die soziale und die biologische Elternschaft würden ohnehin immer stärker auseinanderfallen (S. 114). Körperliche Risiken für die Spenderin sollen „für die Allgemeinheit von Belang“ sein, „weil diese als Solidargemeinschaft für die erheblichen Kosten von Invalidität aufkommen muss“ (S. 118); allerdings müsse es sich um „gravierende Gesundheitsrisiken“ handeln (S. 119). Verwundert registriert Hörnle, dass vertreten wird, nicht die unentgeltliche Spende, wohl aber die entgeltliche „Spende“ verletze die Menschenwürde (S. 120 ff.): Relevant sei einzig, ob die betroffene Person „ohne Autonomiedefizite“ eingewilligt hat (S. 124), und das könne auch der Fall sein, wenn die Eizelle entgeltlich überlassen wird, insbesondere zur Vermeidung der Folgen von Armut. Der Satz, ,wer arm ist, kann nicht frei entscheiden“, sei nicht plausibel (S. 126). - Die meisten Restriktionen verfallen somit dem Verdikt „Rechtsmoralismus“ (S. 127).

Weitgehend vergleichbar argumentiert Schroth zur Nierenlebendspende (S. 205 ff.) Die Beschränkung des Spenderrisikos auf das Operationsrisiko hält er für falsch; etwa bei einer Leberteilspende würden auch höhere Risiken in den Kauf genommen (S. 212. - Aber warum sollte dieses Höchstmaß immer vertretbar sein?). Auch die Subsidiarität der (besser wirkenden!) Lebendspende lässt Schroth nicht durchgehen; denn diese Regelung bewirke nur, dass ein anderer Leidender bei dem Bezug des Leichenorgans hintan gesetzt werde (S. 212). Was das Verbot einer entgeltlichen Spende betrifft, sucht Schroth nach der Ratio bei der Freiwilligkeit der Spende, die er freilich dann nicht für eingeschränkt hält, wenn dem Spender „,ein Rücktrittsrecht bis zum Zeitpunkt der Narkotisierung eingeräumt“ wird (S,. 215); zu verbieten sei nicht die Entgeltlichkeit, sondern wucherische Ausbeutung. - In der Tat, der explantierende Arzt und der implantierende erhalten Honorar, der Empfänger erfährt eine Förderung seiner Gesundheit, aber ausgerechnet den Spender kann man mit Imma- 
teriellem abspeisen? - Auch das erforderliche Näheverhältnis findet keine Gnade vor Schroths Augen. Sein Hauptargument (gegen das BVerfG gerichtet) lautet: „Es gibt keinen inhaltlichen Grund, der plausibel macht, dass die Freiheit des Organspenders in engen persönlichen Beziehungen größer ist, als seine Freiheit gegenüber ihm unbekannten Dritten“ (S. 219). - Mag man bei unentgeltlichen Spenden zweifeln, ob der Unbekannten geltende Altruismus valide durchdacht ist, so kann es sich bei entgeltlichen „Spenden“ doch anders verhalten. Aber vielleicht lassen sich die Gründe für eine Spende nur rechtlich verwalten, wenn sie durch eine gesellschaftliche Institution, eben das Näheverhältnis, gestützt werden.

5.) Eine Diskussion über Paternalismus im Strafrecht kann an den Problemen des Betäubungsmittelstrafrechts nicht vorbeigehen, und diese durchziehen demgemäß den vorliegenden Band von Anfang bis Ende. Birnbacher hält die Bestrafung selbstschädigenden oder -gefährdenden Verhaltens für falsch, da solches Verhalten keinen Tadel verdiene (S. 25). Selbst moralische Pflichten gegen sich selbst schließt Birnbacher „vor dem Hintergrund einer säkularen Aufklärungsmoral“ aus. - Schünemann verfasst eine Philippika gegen „die Strategien des BVerfG zu Aushebelung der rechtsstaatlichen Einwände “ in der Cannabis-Entscheidung (S. 233 ff.); freilich wäre bei seinem Vergleich zwischen Cannabis und Alkohol (S. 234 f.) auch zu berücksichtigen gewesen, was nun einmal kulturell überkommen und deshalb in die Gesellschaft integriert ist; dass auch diese Integration Probleme bringt, ist kein Grund, sich weitere aufzuhalsen.

Auch Woblers und Went lehnen eine Drogenrepression, die aus paternalistischen Erwägungen erfolgt, rundweg ab (S. 292 und passim). Solche Erwägungen seien in der Regel vorgeschoben; der Sache nach gehe es um die Vermeidung sozialer Folgekosten, die Bekämpfung organisierter Kriminalität und „das Interesse, einer Desintegration durch grundsätzlich abweichende Lebensstile entgegenzuwirken“ (S. 302). Das wird am Beispiel des Betäubungsmittelstrafrechts der Niederlande eingehend und eindrucksvoll belegt: Nicht der Schutz der Konsumenten bilde den Grund für die Wende zu einer stärker restriktiven Drogenpolitik seit 1995, vielmehr „die abnehmende gesellschaftliche Toleranz gegenüber den von Drogenabhängigen verursachten Störungen des öffentlichen Friedens (overlast), die um sich greifende organisierte Kriminalität und die ausländische Kritik an der niederländischen Drogenpolitik“ (S. 313, 317, $321 \mathrm{f}$.).

Was die Abwehr abweichender Lebensstile angeht, so ist der Stil der Rauschmittelsüchtigen gewiss nicht vorzugswürdig, und meistens ist auch die Sucht nicht frei gewählt, sondern der Abhängige ist in sie hineingeschlittert. Wer frei sein will, muss die Konsequenzen seines Verhaltens tragen, aber es bleibt doch die Frage, ob eine freiheitliche Gesellschaft verstehbar und akzeptierbar bleibt, wenn sie beliebige Gebrauchsweisen von Willkür nebeneinanderstellt (von einer Integration kann nicht 
einmal annähernd die Rede sein). Ein Gebrauch der Willkür, der in eine Sucht führt, ohne dass dies durch kulturelle Gewinne ausgeglichen würde, desavouiert die Freiheit, ganz unabhängig von den Folgekosten. Der Konsument wäre nicht für die Selbstzerstörung zu tadeln - insoweit kann es bei Birnbachers Feststellung bleiben -, aber vielleicht doch dafür, dass er mit dem exzessiven Gebrauch seiner Willkür die Akzeptation einer freiheitlichen Gesellschaft erschwert.

\section{Schlussbemerkungen}

Wie gezeigt werden sollte, ist in dem Band viel von Freiheit die Rede, freilich weniger von den institutionellen Voraussetzungen, die ein freiheitliches Miteinander erst ermöglichen (immerhin bei Kleinig, von der Pfordten und Yamanaka, Japan, S. 323 ff.). Der Grund dafür liegt in der Bindung an ein Rechtsgüterverletzungsdogma oder ein principle of harm, wodurch die Bestandsbedingungen der Gesellschaft ausgeblendet werden. Die den Band in weiten Teilen durchziehende Paternalismusfeindlichkeit teilt der Rezensent durchaus und räumt auch ein, dass nicht bereits ausgemacht ist, der Institutionenschutz müsse stets durch das Strafrecht erfolgen. Aber allein aus einem mehr oder weniger abstrakten Begriff personaler Freiheit lässt sich keine stabile gesellschaftliche Praxis herleiten.

Nicht auf alle Beiträge des Bandes konnte hier eingegangen werden, ansonsten nicht stets mit der an sich gehörigen Gründlichkeit, und die Auswahl mag von den Präferenzen des Rezensenten zeugen. Jedenfalls sollte deutlich geworden sein, dass ein gewaltiges Thema von vielen Seiten her eingehend und ertragreich behandelt wird; die Lektüre lohnt allemal, auch wenn sich, wie beim Rezensenten, ab und an eine Neigung zum Widerspruch regt und auch wenn ein paar Beiträge bereits vorveröffentlicht sind. Eines ist freilich ärgerlich: Die Sequenz der Tagungsbeiträge nach Rede, Ergänzung und Gegenrede wird zerstört, weil sich die Herausgeber zu einer alphabetischen Ordnung nach den Namen der Verfasser und Verfasserinnen entschlossen haben, was zu „Sprüngen“ bei der Lektüre nötigt. Freilich ist diese Kritik sogleich zu relativieren; denn selbstständig lesenswert sind sämtliche Beiträge, auch die hier nicht erwähnten. 